\title{
The effects of different post-thawed culture periods on clinical outcomes in frozen embryo transfer cycle: a retrospective study
}

\author{
Yuhu $\mathrm{Li}^{1}$, Xuexiang $\mathrm{Cai}^{1}$, Bo $\mathrm{Ma}^{1}$, Ning $\mathrm{LI}^{1}$, and liuguang zhang ${ }^{1}$ \\ ${ }^{1}$ Affiliation not available
}

April 21, 2021

\begin{abstract}
Objective To evaluate the effects of different post-thawed culture periods on the clinical outcomes Design A retrospective study. Setting Two IVF centers. Population Women undergoing first cleavage-stage embryo transfer in frozen-thawed cycles. Methods 9832 FET cycles were divided into three groups according to female age: $<35,35-39$ and $>39$ years, and two groups depending on post-thawed culture period: short and long culture groups. The long culture group divided into three groups depending on blastomere growth number: [?] 2, one [?] 2 and the other $>2$, and $>2$ groups. Main Outcome Measures Implantation rate (IR), clinical pregnancy rate (CPR), multiple pregnancy rate (MPR), live birth rate (LBR) and neonatal characteristics. Results Long post-thawed culture caused a significant increase in the IR, CPR, MPR and LBR (P/CI = 0.002/1.034-1.162, 0.027/1.011-1.194, 0.028/1.014-1.255 and 0.001/1.054-1.245 respectively), and blastomere growth number had a significant effect on IR, CPR, MPR and LBR $(\mathrm{P}=0.000,0.000,0.000$ and 0.000 respectively). No significant differences were present in neonatal characteristics between the two post-thawed culture groups. Singleton group had a higher average gestational age and birthweight as well as a lower cesarean section rate, preterm labor rate and low birthweight rate. Conclusions Long post-thawed culture was associated with higher IR, CPR, MPR and LBR, and transferring a well-developed embryo after long post-thawed culture might be a viable embryo transfer strategy to decrease MPR while maintaining CPR and LBR. Funding None Keywords Post-thawed culture, blastomere growth, neonatal characteristics, live birth rate.
\end{abstract}

\section{Introduction}

With the development of vitrification technology, the survival and intact cell rates are increasing and the implantation, clinical pregnancy and live birth rates of the vitrification are similar to the fresh. ${ }^{1-3}$ Embryo cryopreservation makes it possible to conserve surplus embryos for further use, and increases cumulative livebirth rates with one oocyte retrieval operation. The advantages of frozen-thawed embryo transfer (FET) are not only decreasing the rate of ovarian hyperstimulation syndrome (OHSS), but also providing a more favorable intrauterine environment for embryo implantation and placentation by avoiding the supraphysiologic condition that occurs after ovarian stimulation. ${ }^{4-7}$ Hence, the proportion of FET is increasing in assisted reproductive technologies. The factors affecting the outcome of FET are female age, embryo characteristics, endometrial preparation protocol, number of embryos transferred and so on. 8,9

Currently, the two most common procedures to select embryos for FET differ in the duration of the postthawed culture: one relies upon the observation of blastomere survival after thawing, requiring a short culture; another is dependent on the observation of blastomere proliferation, requiring a long culture, generally overnight. Some studies indicated that cleavage stage embryos with low blastomere number had significantly decreased developmental potential and pregnancy rate, ${ }^{10-12}$ and extended culture of these embryos to blastocysts can resulted in a similar clinical pregnancy rate as that of blastocysts derived from embryos with [?] 7 blastomeres. ${ }^{12,} 13$ The influence of post-thawed culture for about $18 \mathrm{~h}$ on the outcome of FET is still controversial, ${ }^{12,14,15}$ and the reasons may be related to the different sample sizes, inclusion 
criteria, embryo freezing methods, embryo quality and age distribution of patients in different studies. Previous studies focused on the comparison between short culture and long culture, while ignoring the effect of age and embryo quality on implantation and live birth rates. In this study, a retrospective analysis improved sample size (containing 9832 FET cycles), age distribution (divided into three groups: $<35,35-39$ and $>$ 39 ) and embryo quality (good-quality embryos) to evaluate the influence of long post-thaw culture period on pregnancy and clinical outcomes.

\section{Methods}

\section{Participants}

This retrospective study was carried out from January 2016 to December 2019 at the Center of Reproductive Medicine of Mali Hospital, Haikou, China and the Hospital of Shenzhen Immigration Inspection, Shenzhen, China. This study included the first frozen-thawed cleavage-stage embryo transfer (FET) cycle of women who underwent IVF cycles with or without ICSI (Intracytoplasmic Sperm Injection) with an indication of tubal, male, or unexplained infertility. Women with a diagnosis of a congenital or acquired uterine abnormality, such as a uterine malformation, adenomyosis, submucous myoma, uterine fibroids or intrauterine adhesions were excluded from this study. The two post-thawed cleavage-stage embryos for each FET cycle were goodquality embryos that had 7-9 equal size or roughly equal size blastomeres and fragments $<20 \%$, and derived from normally fertilized oocytes. Thawed embryos with damaged blastomeres and the transfer of one or three embryos were excluded.

\section{Study design}

The 9832 FET cycles were divided into three groups according to female age: < 35, 35-39 and > 39 years, and two groups depending on their post-thawed culture period: short culture $(2-3 \mathrm{~h})$ group $(\mathrm{S})$ and long culture (18-20 h) group (L). The long culture group divided into three groups depending on the blastomere growth number: [?] 2 group (blastomere growth number of each transferred embryo was [?] 2), one [?] 2 and the other $>2$ group (blastomere growth number of the two transferred embryos was that one was [?] 2 and the other was greater than $>2$ ) and $>2$ group (blastomere growth number of each transferred embryo was $>2$ ).

Clinical outcomes included implantation rate (IR), clinical pregnancy rate (CPR), multiple pregnancy rate (MPR), abortion rate (AR), live birth rate (LBR), ectopic pregnancy rate (EPR), gestational age, preterm labor rate, mode of delivery, sex ratio, congenital malformation, low birthweight rate and birthweight were compared. IR was defined as the number of gestational sacs on ultrasound divided into the total number of transferred blastocysts. Clinical pregnancy was considered as a gestational sac seen by vaginal ultrasound scan by 6-7 weeks of gestation, while multiple pregnancy was described as having more than one gestational sac. Meanwhile, abortion was the spontaneous cessation of a clinical pregnancy, and live birth was defined as delivery of a live fetus at gestational age [?] 24 weeks.

\section{Embryo culture, freezing and thawing protocols}

Fertilization and embryo culture were carried out in Quinn's IVF sequential medium suite (Quinn's, SAGE, USA) with adding $10 \%$ human serum substitute (Quinn's, SAGE, USA) in $5 \% \mathrm{O}_{2}, 5 \% \mathrm{CO}_{2}, 90 \% \mathrm{~N}_{2}$ and saturated humidity. Follicles were aspirated transvaginally under ultrasound guidance at $36 \mathrm{~h}$ post hCG injected. Cumulus-oocyte complexes and sperm were mixed at 39-40 h post hCG injected. For ICSI, the cumulus cells were removed enzymatically and the sperm was injected at about $42 \mathrm{~h}$ after hCG injected. The fertilization results were evaluated $16-18 \mathrm{~h}$ after fertilization and embryos were cultured individually in 25 $\mu \mathrm{l}$ microdroplet of cleavage medium covered with oil. Cleavage-stage embryos were scored and graded every day for blastomere number, cleavage plane and degree of fragmentation. Available embryos were vitrified using vitrification media (Kitazato Biopharma, Shizuoka, Japan) on day 3 after egg collection (D3). Briefly, embryos were transferred from the culture medium to the center of the equilibration solution surface and remained in the media for about 7 minutes at room temperature. Then, the embryos were moved into vitrification solution and blown and inhaled at different positions. After 40-50 s in this solution, the embryos 
were placed on the Cryotop (Kitazato Biopharma, Shizuoka, Japan) and rapidly placed into liquid nitrogen. Embryos were thawed using vitrification media (Kitazato Biopharma, Shizuoka, Japan). Briefly, Cryotop was taken rapidly from liquid nitrogen and immersed in a $300 \mu \mathrm{l}$ droplet of thawing solution (TS) at 37. At the end of 1 minute, the embryos were transferred to a $300 \mu$ d droplet of diluent solution (DS) at room temperature for 3 minutes. Then, the embryos were shifted to $300 \mu$ l droplets of washing solution 1 (WS1) and washing solution 2 (WS2) at room temperature for 5 minutes, respectively. Finally, the embryos were transferred to a $30 \mu \mathrm{l}$ droplet of blastocyst medium and cultured for $2-3 \mathrm{~h}$ or $18-20 \mathrm{~h}$ for transfer.

\section{Endometrial preparation}

Natural cycle and artificial cycle were used for FET. Natural cycle was conducted either by administration of hCG for planning the transfer or by detecting the spontaneous LH peak, and cleavage-stage embryos were transferred on the third day after ovulation. In terms of the artificial cycle, $3.75 \mathrm{mg}$ per day of oral oestradiol valerate was started on days 2-3 of the menstrual cycle, and the dose of estradiol valerate was adjusted according to the endometrial thickness monitored by ultrasound. When the endometrial thickness reached [?] $7 \mathrm{~mm}, 40 \mathrm{mg} / \mathrm{d}$ progesterone was injected 3 days prior to frozen embryos transfer. If pregnancy was achieved, luteal phase support was continued until 11 weeks' gestation.

\section{Statistical analyses}

Statistical analyses were performed with SPSS 22.0 (IBM, Armonk, USA). Quantitative variables were presented as means \pm standard deviations and categorical variables were expressed as frequencies and percentages. The data were analyzed using the Chi-squared test, one-way analysis of variance (ANOVA) or Bonferroni test as appropriate. Logistic regression analysis was performed to explore the effects of postthawed culture period on live birth after controlling for potential confounders including female age, female BMI, endometrial preparation, type of infertility and duration of infertility. A two-sided $P$-value $<0.05$ was considered to be statistically significant.

\section{Results}

\section{Patient and cycle characteristics}

From January 2016 to December 2019, 9832 frozen-thawed D3 embryo transfer cycles from a total of 23366 were analyzed, including 6013 short culture cycles (2-3 h of post-thawed culture) and 3819 long culture cycles (18-20 h of post-thawed culture) (Figure S1). The female age, duration of infertility and body-mass index were $20-45$ years, $0-25$ years and $12-42 \mathrm{~kg} / \mathrm{m}^{2}$ respectively. The detailed cycle characteristics of the different groups are shown in Table S1. Significant differences of average age between L and S groups were observed in the $<35,35-39$ and total age groups $(P / \mathrm{CI}=0.001 / 0.112-0.416,0.046 /-0.218-0.002,0.000 / 0.226-0.630$ respectively), and the differences of body-mass index, natural cycle rate, primary infertility rate and duration of infertility were not significant in all age groups.

\section{Pregnancy and clinical outcomes}

Table 1 presents the effects of different post-thawed culture periods on pregnancy and clinical outcomes. The results indicated that the post-thawed culture period had significant effects on pregnancy and clinical outcomes. In total age group, IR, CPR, MPR and LBR of L group were observed to be higher than that of S group $(P / \mathrm{CI}=0.002 / 1.034-1.162,0.027 / 1.011-1.194,0.028 / 1.014-1.255$ and $0.001 / 1.054-1.245$ respectively $)$, while EPR was significant high in short culture group $(P / \mathrm{CI}=0.029 / 1.043-1.983)$ and AR was no significant difference between the two groups $(P / \mathrm{CI}=0.172 / 0.962-1.260)$. In the $<35$ year groups, IR, CPR and LBR were significant high in the $\mathrm{L}$ group compared with these in the $\mathrm{S}$ group $(P / \mathrm{CI}=0.001 / 1.061-1.233$, 0.004/1.056-1.332, 0.000/1.118-1.396 respectively), while AR and EPR were observed to be higher in the $\mathrm{S}$ group than these in the $\mathrm{L}$ group $(P / \mathrm{CI}=0.036 / 1.015-1.472,0.002 / 1.272-2.864$ respectively). Moreover, MPR was high of $\mathrm{L}$ group than that of $\mathrm{S}$ group $(P / \mathrm{CI}=0.071 / 0.990-1.280)$. In the 35-39 age group, IR, CPR, MPR, EPR and AR were similar between groups L and S, while LBR was observed to be higher in the $\mathrm{L}$ group than these in the $\mathrm{S}$ group $(P / \mathrm{CI}=0.039 / 1.010-1.387)$. In the $>39$ age group, there was no significant difference on IR, CPR, MPR, AR, EPR and LBR between S and L groups. Table 1 also displays 
the effect of female age on pregnancy and clinical outcomes. The results indicated that IR, CPR, MPR and LBR decreased significantly and AR increased significantly ( $P$ values were all less than 0.001$)$ with age in the $\mathrm{S}$ and $\mathrm{L}$ groups, while EPR had not significant difference with age.

Table 2 shows the effect of blastomere growth number after long culture period of thawed D3 embryo on pregnancy and clinical outcomes. The results indicated that blastomere growth number had a significant effect on IR, CPR and LBR in all age groups ( $P$ values were all less than 0.001$)$, and the faster the embryo developed, the better outcome was found. MPR significantly increased with the increase of blastomere growth number in $<35,35-39$ and total age groups ( $P=0.000,0.001$ and 0.000 respectively), while MPR was similar in $>39$ age group $(P=0.235)$. AR was similar in $<35$ and $>39$ age groups $(P=0.353$ and 0.948$)$ and the rates of [?] 2 blastomeres groups significantly decreased in 35-39 and total age groups $(P=0.028$ and 0.050). Moreover, no significant differences were found in EPR among the four age groups. The IR, CPR and LBR of same blastomere growth number groups decreased significantly and AR increased significantly with age ( $P$ values were all less than 0.001$)$.

A logistic regression model for predicting live birth is given in Table 3. Variables that were statistically significant in Table S1, or those that were deemed to be clinically significant, were included in order to build the model. This model indicated that the most significant predictors for live birth were female age, type of infertility and culture period (D3/D4). This model shows that the most significant predictors for live birth were female age, secondary infertility and long post-thawed culture period $(P / \mathrm{CI}=0.00 / 0.89-0.91$, 0.00/1.06-1.28 and 0.00/1.11-1.32 respectively).

\section{Neonatal characteristics}

Table 4 presents the neonatal characteristics of singletons and twins in the different post-thawed culture period groups. No significant differences were present in regard to gestational age, preterm labor rate, birthweight, low birthweight rate, cesarean section rate, sex ratio and congenital malformation rate between the two groups. The results also indicated that the singleton had a higher average gestational age and birthweight as well as a lower cesarean section rate, preterm labor rate and low birthweight rate than the twins in the groups $\mathrm{S}$ and $\mathrm{L}$ ( $P$ values were all less than 0.001$)$.

\section{Discussion}

\section{Main Findings}

In this retrospective study, the results demonstrated long post-thawed culture could be causing a significant increase in the IR, CPR, MPR and LBR. Then transferred embryos with more blastomere growth had a higher IR, CPR, MPR and LBR. The study also indicated that the neonatal characteristics between L and $\mathrm{S}$ groups were similar, and the twin group had a lower average gestational age and birthweight as well as a higher cesarean section rate, preterm labor rate and low birthweight rate than the singleton group.

\section{Strengths and Limitations}

Compared with previous studies, ${ }^{14,} 15$ the study had some advantages in its design that afforded strong confidence on the results. Firstly, the study was based on a large sample including 9832 FET cycles. Then, in order to eliminate the effects of the embryo quality on the clinical outcomes in this study, we selected the FET cycles that the two embryos after thawed were good-quality embryos. Moreover, the study divided the female age into three groups to decrease the effects of female age on clinical outcomes. Furthermore, the study selected first FET cycle and excluded FET cycles with abnormal uterine to ensure the accuracy of data. One limitation of this study was that patients' characteristic of the number of oocytes from the OPU was not mentioned.

\section{Interpretation}

Female age and quality of transferred embryos were usually accepted as the two most important causes of bias in assay comparisons. In the study, IR, CPR, MPR and LBR were decreased significantly and AR increased significantly with age. The differences of IR, CPR, AR, LBR and EPR between groups S and L 
were significant in $<35$ age group, while the differences of these rates were almost similar in the groups 35-39 and $>39$. These results indicated female age was a critical impact factor for clinical outcomes, and different female age distribution of patients in different studies may be connected with different study results. Previous studies demonstrated that D3 embryos with [?] 6 blastomeres were associated with low IR, CPR, and LBR and high AR compared with those with $>6$ blastomeres. ${ }^{10,12,16}$ So, the quality of transferred embryos may be another factor contributing to the discrepancy in different studies. One limitation of this study was that patients' characteristic of the number of oocytes from the OPU was not mentioned. Because, the limited number of oocytes available after ovarian stimulation was usually associated with advanced maternal age and/or premature ovarian insufficiency, and dramatically decrease of the number and implantation potential of oocytes were together with a dramatic pick up of aneuploidy rates over $70 \% .{ }^{17}$ In addition, women with advanced maternal age had higher risk for miscarriage (over 40\%) as well as drastically lower rate of clinical pregnancy. ${ }^{17}$ Therefore, the limited number of oocytes available after ovarian stimulation may be an important risk factor affecting the LBR.

With the development of in vitro culture technology, elective single blastocyst transfer has become a tendency, and this practice has led to a remarkable increase in LBR and decrease in MPR. ${ }^{18,}{ }^{19}$ However, the failure to form blastocysts in patients with few day 3 cleavage stage embryos would lead to an increase in cycle cancelation. Therefore, a cleavage transfer policy is still adopted in most centers, as this practice can be used for a wider range of infertility patients. Two or three cleavage stage embryos are transferred in traditional cleavage-stage transfer policy which increases the CPR with the occurrence of high MPR at the same time. Previous study found that morula embryos, developed from post-thawed cleavage embryos, resulted in a higher IR, CPR and LBR than non-morula embryos in FET cycles, ${ }^{20}$ and extended culture of D3 embryos with [?] 6 blastomeres to blastocysts, particularly 6-cell embryos, resulted in a similar clinical pregnancy rate as that of blastocysts derived from D3 embryos with [?] 7 blastomeres. ${ }^{12}$ The CPR, IR, and LBR were similar in the elective single morula embryo transfer group and elective single blastocyst embryo transfer group. ${ }^{21,} 22$ A trend toward higher clinical and ongoing pregnancy rates was noted after day 4 embryo transfers when compared with day 3 embryos in ET cycles. ${ }^{23}$ The development of the fair embryos transferred after 20-22 $\mathrm{h}$ in the culture had a highly positive impact on the pregnancy rate. ${ }^{24}$ Our study also found that transferred embryos with more blastomere growth had a higher IR, CPR, MPR and LBR. The higher MPR in the $>2$ group $(47.5 \%)$ caught our attention and suggested that the embryos with more blastomere growth number were associated with better embryo viability. Even if two embryos with less blastomere growth number ([?] 2 group) were transferred, the MPR remained high (29.0\%). Therefore, transferring a well-developed embryo after long post-thawed culture period may be a viable embryo transfer strategy to decrease MPR and cycle cancellation rate while maintaining CPR and LBR. The reasons of higher IR in the L group may be that embryos with hardened zona pellucida have a relatively long time to hatch out and implant in the synchronous endometrium. Moreover, the development of embryos in vitro lags behind that in vivo, so earlier thawing is beneficial to synchronize the development of embryos and endometrium.

Neonatal characteristics of gestational age, preterm birth rates, cesarean section rate, birthweight, low birthweight rate, sex ratio and congenital malformation rate were similar in the long culture period group compared with these in the short culture period group. These results indicated that long post-thawed culture period might be harmless to the clinical outcomes of FET compared with short post-thawed culture period. Multiple pregnancies are considered the most significant adverse event associated with assisted reproductive technologies and is linked to an increased risk of maternal and neonatal morbidity. The study also showed the twin group had a lower average gestational age and birthweight as well as a higher cesarean section rate, preterm labor rate and low birthweight rate than the singleton group. Therefore, elective single welldeveloped embryo transfer after long post-thawed culture period or elective single blastocyst transfer after blastocyst culture are recommended to decrease the risk of maternal and neonatal morbidity caused by multiple pregnancy.

\section{Conclusion}

The study suggests that long post-thawed culture period can be causing an increase in the IR, CPR, MPR 
and LBR, and transferred embryos with more blastomere growth number after long post-thawed culture period has a higher IR, CPR, MPR and LBR. Therefore, transferring a well-developed embryo after long post-thawed culture period may be a viable embryo transfer strategy to decrease MPR while maintaining CPR and LBR.

\section{Acknowledgements}

The authors would like to thank all participants and staff at IVF Center, Mali Hospital, Haikou, China and the Hospital of Shenzhen Immigration Inspection, Shenzhen, China.

\section{Disclosure of Interests}

The authors declare that they have no conflict of interest.

\section{Contribution to Authorship}

The study was designed by YHL, BM and LGZ. XXC, NL and YHL were involved in planning and managing the data collection. YHL and BM were involved in the statistical analysis and wrote the manuscript with support and critical review from all authors.

\section{Details of Ethical Approval}

Ethical approval was granted by the Ethics Committee of Mali Hospital on the March 8, 2020 (NO. 01/MLYY/2020).

\section{Funding}

None.

\section{Reference}

1 Kolibianakis EM, Venetis CA, Tarlatzis BC. Cryopreservation of human embryos by vitrification or slow freezing: which one is better? Curr Opin Obstet Gynecol2009; 21: 270-4.

2 Rienzi L, Gracia C, Maggiulli R, LaBarbera AR, Kaser DJ, Ubaldi FM, et al. Oocyte, embryo and blastocyst cryopreservation in ART: systematic review and meta-analysis comparing slow-freezing versus vitrification to produce evidence for the development of global guidance. Hum Reprod Update 2017; 23: 139-55.

3 Shi Y, Sun Y, Hao C, Zhang H, Wei D, Zhang Y, et al. Transfer of Fresh versus Frozen Embryos in Ovulatory Women. N Engl J Med 2018; 378: 126-36.

4 Evans J, Hannan NJ, Edgell TA, Vollenhoven BJ, Lutjen PJ, Osianlis T, et al. Fresh versus frozen embryo transfer: backing clinical decisions with scientific and clinical evidence. Hum Reprod Update 2014; 20: $808-21$.

5 Roque M, Haahr T, Geber S, Esteves SC, Humaidan P. Fresh versus elective frozen embryo transfer in IVF/ICSI cycles: a systematic review and meta-analysis of reproductive outcomes. Hum Reprod Update 2019; 25: 2-14.

6 Wong KM, Van WM, Mol F, Repping S, Mastenbroek S. Fresh versus frozen embryo transfers in assisted reproduction. Cochrane Database Syst Rev 2017; 3: CD011184.

7 Bourdon M, Maignien C, PocateCK, Plu Bureau G, Marcellin L, Patrat C, et al. The freeze-all strategy after IVF: which indications? Reprod Biomed Online 2020; 42: 529-45

8 Veleva Z, Orava M, NuojuaHS, Tapanainen JS, Martikainen H. Factors affecting the outcome of frozenthawed embryo transfer. Hum Reprod 2013; 28: 2425-31.

9 Salumets A, Suikkari AM, Makinen S, Karro H, Roos A, Tuuri T. Frozen embryo transfers: implications of clinical and embryological factors on the pregnancy outcome. Hum Reprod 2006; 21: 2368-74. 
10 Kong X, Yang S, Gong F, Lu C, Zhang S, Lu G, et al. The relationship between cell number, division behavior and developmental potential of cleavage stage human embryos: atime-lapse study. PLoS One 2016; 11: e0153697.

11 Van LL, Van WM, Van VF, Bossuyt P, Repping S. Selection of embryos for transfer in IVF: ranking embryos based on their implantation potential using morphological scoring. Reprod Biomed Online 2014; 29: $222-30$.

12 Li B, Huang J, Li L, He X, Wang M, Zhang H, et al. Improving the clinical outcomes by extended culture of day 3 embryos with low blastomere number to blastocyst stage following frozen-thawed embryo transfer. Arch Gynecol Obstet2020; 303: 573-80.

13 Zhao P, Li M, Lian Y, Zheng X, Liu P, Qiao J. The clinical outcomes of day 3 4-cell embryos after extended in vitro culture. J Assist Reprod Genet 2015; 32: 55-60.

14 Rato ML, Gouveia-Oliveira A, Plancha CE. Influence of post-thaw culture on the developmental potential of human frozen embryos. J Assist Reprod Genet 2012; 29: 789-95.

15 Guo L, Luo C, Quan S, Chen L, Li H, Guo Y, et al. The outcomes of different post-thawed culture period in frozen-thawed embryo transfer cycle. J Assist Reprod Genet 2013; 30: 1589-94.

16 Zhang S, Lu C, Lin G, Gong F, Lu G. The number of blastomeres in post-thawing embryos affects the rates of pregnancy and delivery in freeze-embryo-transfer cycles.J Assist Reprod Genet 2009; 26: 569-73.

17 Milachich T, Shterev A. Are there optimal numbers of oocytes, spermatozoa and embryos in assisted reproduction? JBRA Assist Reprod 2016; 20: 142-9.

18 Wei D, Liu JY, Sun Y, Shi Y, Zhang B, Liu JQ, et al. Frozen versus fresh single blastocyst transfer in ovulatory women: a multicentre, randomised controlled trial.Lancet 2019; 393: 1310-8.

19 Zhu Q, Lin J, Gao H, Wang N, Wang B, Wang Y. The association between embryo quality, number of transferred embryos and live birth rate after vitrified cleavage-stage embryos and blastocyst transfer. Front Physiol 2020; 11: 1-7.

20 Hui D, Han X, Wang X, Ren W, Lei X, Liu J, et al. Morula transfer achieves better clinical outcomes than post-thawed cleavage embryos after overnight culture in frozen embryo transfer (FET) cycles. $J$ Assist Reprod Genet 2020; 37: 945-52.

21 Kang SM, Lee SW, Jeong HJ, Yoon SH, Koh MW, Lim JH, et al. Clinical outcomes of elective single morula embryo transfer versus elective single blastocyst embryo transfer in IVF-ET. J Assist Reprod Genet 2012; 29: 423-8.

22 Li RS, Hwu YM, Lee RK, Li SH, Lin MH. Day 4 good morula embryo transfer provided compatible live birth rate with day 5 blastocyst embryo in fresh IVF/ET cycles. Taiwan J Obstet Gynecol 2018; 57: 52-7.

23 Pantos K, Makrakis E, Chronopoulou M, Biba M, Perdikaris A, Dafereras A. Day 4 versus day 3 embryo transfer: a prospective study of clinical outcomes. Fertil Steril 2008; 89: 573-7.

24 Haas J, Meriano J, Bassil R, Barzilay E, Casper RF. Prolonged culture of blastocysts after thawing as a tool for improving prediction of success. J Assist Reprod Genet 2018; 35: 2195-9.

\section{Hosted file}

Table1-4.pdf available at https://authorea.com/users/409218/articles/518983-the-effects-ofdifferent-post-thawed-culture-periods-on-clinical-outcomes-in-frozen-embryo-transfercycle-a-retrospective-study 